\title{
Comparative mechanical behavior of dentin enamel and dentin ceramic junctions assessed by speckle interferometry (SI)
}

\author{
Michel Fages ${ }^{a, *}$, Pierre Slangen ${ }^{b}$, Jacques Raynal ${ }^{a}$, Stephane Corn ${ }^{b}$, Kinga Turzo ${ }^{c}$, \\ Jacques Margerit ${ }^{a}$, Frédéric J. Cuisinier ${ }^{a}$ \\ a EA4203, Department of Biologic Sciences, University of Montpellier I, Montpellier, France \\ ${ }^{\mathrm{b}}$ Ecole des Mines d'Alès, I.L.O.A., Alès, France \\ c Department of Prosthodontic and Oral Biology, University of Szeged Dental School, Szeged, Hungary
}

\section{A R T I C L E I N F O}

Article history:

Received 23 October 2011

Received in revised form 3 May 2012

Accepted 18 May 2012

Available online $\mathrm{xxx}$

Keywords:

Dentin enamel junction (DEJ)

Dentin ceramic junction (DCJ)

Speckle interferometry (SI)

Tooth crown

Ceramic crown

\begin{abstract}
A B S T R A C T
Objective. The dentin-enamel junction (DEJ) plays a crucial role in dental biomechanics; however, little is known about its structure and mechanical behavior. Nevertheless, natural teeth are a necessary model for prosthetic crowns. The mechanical behavior of the natural DEJ and the dentin ceramic junction (DCJ) manufactured with a CAD-CAM system are compared. Methods. The reference samples undergo no modification, while the experimental samples were drilled to receive a cemented feldspathic ceramic crown. Longitudinally cut samples were used to achieve a planar object observation and to look "inside" the tooth. A complete apparatus enabling the study of the compressive mechanical behavior of the involved tooth by a non-contact laser speckle interferometry (SI) was developed to allow nanometric displacements to be tracked during the compression test.

Results. It is observed that the DEJ acted as a critical zone accommodating the movement between dentin and enamel. A smooth transition occurs between dentin and enamel. In the modeled prosthetic, the same kind of accommodation effects also occurs, but with a steeper transition slope between dentin and ceramic.

Significance. On the natural tooth, the stress accommodation arises from a differential behavior between enamel and dentin from the DEJ. In the ceramic crown, the cemented dentin-ceramic junction should play this role. This study demonstrates the possible realization of prosthetic crown reconstructions approaching biomechanical behaviors.

(c) 2012 Academy of Dental Materials. Published by Elsevier Ltd. All rights reserved.
\end{abstract}

\section{Introduction}

The dentin-enamel junction (DEJ) in teeth is the zone between two distinct calcified tissues with very different biomechanical properties: enamel and dentin [1]. Enamel is hard and brittle and envelops the softer dentin. The enamel and dentin work together during the many load cycles experienced by the tooth over its lifetime. Generally, interfaces between materials with dissimilar elastic and mechanical properties represent "weak links" in a structure; however, the DEJ acts to successfully transfer applied loads (e.g., masticatory or impact) from the enamel to the dentin and inhibits enamel cracks from propagating into the dentin and causing tooth fracture $[2,3]$.

\footnotetext{
* Corresponding author at: 11 Avenue Celestin Arnaud, 34110 La Peyrade, France. Tel.: +33 6848557 15; fax: +33467486092.

E-mail address: mifages@wanadoo.fr (M. Fages).

0109-5641/\$ - see front matter @ 2012 Academy of Dental Materials. Published by Elsevier Ltd. All rights reserved. http://dx.doi.org/10.1016/j.dental.2012.05.006
} 
The DEJ appears as a discrete line when visualized microscopically and is thought to represent the original position of the basement membrane of the ameloblasts and odontoblasts where they coincide in the embryological tooth bud [4]. In human enamel and dentin, fatigue damage is the end result of extreme loads and is frequently associated with pathology or extensive wear. The fracture-resistant properties of the DEJ are believed to originate from a gradual change in microstructure and in the properties of dentin and enamel rather than from the abrupt transition between the two dissimilar materials $[5,6]$. Wang and Weiner suggested that the DEJ is one of the working sites of the tooth during mastication [7]. Imbeni et al. [1] believe that collagen fibrils perpendicular to the interface constitute the key reinforcing mechanism at the DEJ, thus explaining why so few cracking events cause delamination when they impinge on the DEJ. Zaslansky et al. $[8,9]$ highlighted the importance of the DEJ as the binding interface between enamel and dentin. They have shown that adjacent to the DEJ is a $200-300 \mathrm{~mm}$-thick zone of dentin of a much lower stiffness (compression elastic modulus) than the bulk of the dentin in the tooth.

Restorations that are all ceramic require proper adhesive bonding on the dentin to achieve their required life expectancy. All-ceramic restorations are made with feldspathic or zirconia ceramics. The strongest ceramics have a fracture toughness of at least $3.0 \mathrm{MPa} \mathrm{m}^{1 / 2}$ [10], which is relatively close to the enamel fracture toughness of $1.3 \mathrm{MPa} \mathrm{m}^{1 / 2}$, in a direction perpendicular to the enamel rods [8]. Nevertheless, fractures of the ceramic part of all-ceramic crowns are difficult to prevent, and crack growth is a significant problem [11]. This phenomenon can be explained by the absence of a stress accommodation zone. The natural stress accommodation zone of 200-300 $\mu \mathrm{m}$-thick dentin has a much lower stiffness than the bulk of the dentin core [8].

Bonding agents must be selected very carefully because they determine not only the adhesion but also the ultimate strength of full-ceramic crowns [12-14]; therefore, it is important to compare the mechanical behavior of natural teeth and of the all-ceramic crown cemented on dentin. Instead of "cement joint", we will use the term "dentin-ceramic junction" (DCJ).

We applied compressive forces representative of those occurring in the oral cavity on natural teeth and all-ceramic crowns, and we determine the relative movement of enamel and dentin, or ceramic crown and dentin, respectively.

\section{Materials and methods}

\subsection{Natural teeth}

Intact lower first premolars free of caries were stored in physiological serum after having been extracted as part of the routine orthodontic treatment of young healthy adolescent patients (aged $<18$ ). Five sets of two samples each (one natural tooth and one prosthetic tooth) were amassed. Right and left premolars from the same patient were used. One was kept intact, and the other was prepared to receive the prosthetic crown.

\subsection{Prosthetic crowns}

We employed the Cerec $3 \mathrm{D}^{\circledR}$ (Sirona Dental System ${ }^{\circledR}$, Bensheim, Germany) CAD/CAM (computer aided design/computer aided manufacturing) unit to manufacture each prosthetic crown as a clone of the opposite tooth using the reproduction capability of the Cerec ${ }^{\circledR}$ software V2.80. This CAD/CAM system is composed of two distinct units: the optical imprint recording also allowing the CAD, and the milling unit using the CAD data to manufacture the sample out of a ceramic block. The software was set to give a dento-prosthetic spacing of $100 \mu \mathrm{m}$ and a peripheral joint of $40 \mu \mathrm{m}$ for a total thickness of $800 \mu \mathrm{m}$. Optical imprints, of the prepared tooth and of the opposite tooth, were recorded, and the Cerec $\mathrm{MC}^{\circledR}$ machine milled the prosthetic crowns [15]. The whole cloning process is presented in Fig. 1.

The Vita MarkII ${ }^{\circledR}$ (Vita Zahnfabrik, Bad Säckingen, Switzerland) ceramic blocks of albite-enriched feldspathic ceramic were used. Their abrasion coefficient is close to that of natural dental enamel. After milling, the extrados were glazed (Azkent ${ }^{\circledR}$, Vita Zahnfabrik, Bad Säckingen, Switzerland).

The crowns were cemented onto the prepared teeth using Relyx Unicem ${ }^{\circledR}$ adhesive cement (3MESPE Dental Division, St. Paul, MN, USA) following the standard clinical protocol of illumination of each side of the crown for $4 \mathrm{~s}$ at $3000 \mathrm{~mW} / \mathrm{cm}^{2}$ with a Swissmaster Light ${ }^{\circledR}$ lamp (E.M.S., Nyons, Switzerland).

\subsection{Specimen preparation}

After extraction, the teeth were disinfected and stored in physiological serum with traces of chloroform. The teeth were longitudinally cut in the vestibular-lingual orientation, and one of the two resulting parts was removed with a diamond disc. Longitudinal cuts have been used to allow planar observation and to appreciate the different behaviors inside the tooth of the natural DEJ and of the DCJ interfaces. The tooth was then glued into the sample holder with a layer of Araldite ${ }^{\circledR}$ (Hunstman Advanced Materials, The Woodlands, Texas, USA). The sample holders have been cast in chromium cobalt using the imprint of a root. The mechanical stability of the specimen holders was validated by a speckle interferometry (SI) experiment.

\subsection{Loading system}

The compression test device fulfills the high sensitivity of speckle interferometry and copes with the rigid body motions of the whole system. The sample tooth cemented in the sample holder was placed under the force transducer (Model 31, Honeywell International, Morriston, NJ, USA). This mid-range precision miniature load cell is slowly translated vertically by the motor $\left(\mathrm{M}-235^{\circledR}\right.$, PI. Karlsruhe, Germany). The system can generate a force-driven displacement (C-862 Mercury PI, Karlsruhe, Germany) or simply a user's displacement. The compression apparatus communicates with the computer through a NI USB-6251 port (National Instruments, Austin, TX, USA) and is interfaced with an in-house LabView program. The entire mechanical system was bolted onto the holographic table top (Newport, Irvine, CA, USA). Very small displacement steps, as small as $1.6 \mathrm{~nm}$, can theoretically be achieved. The 

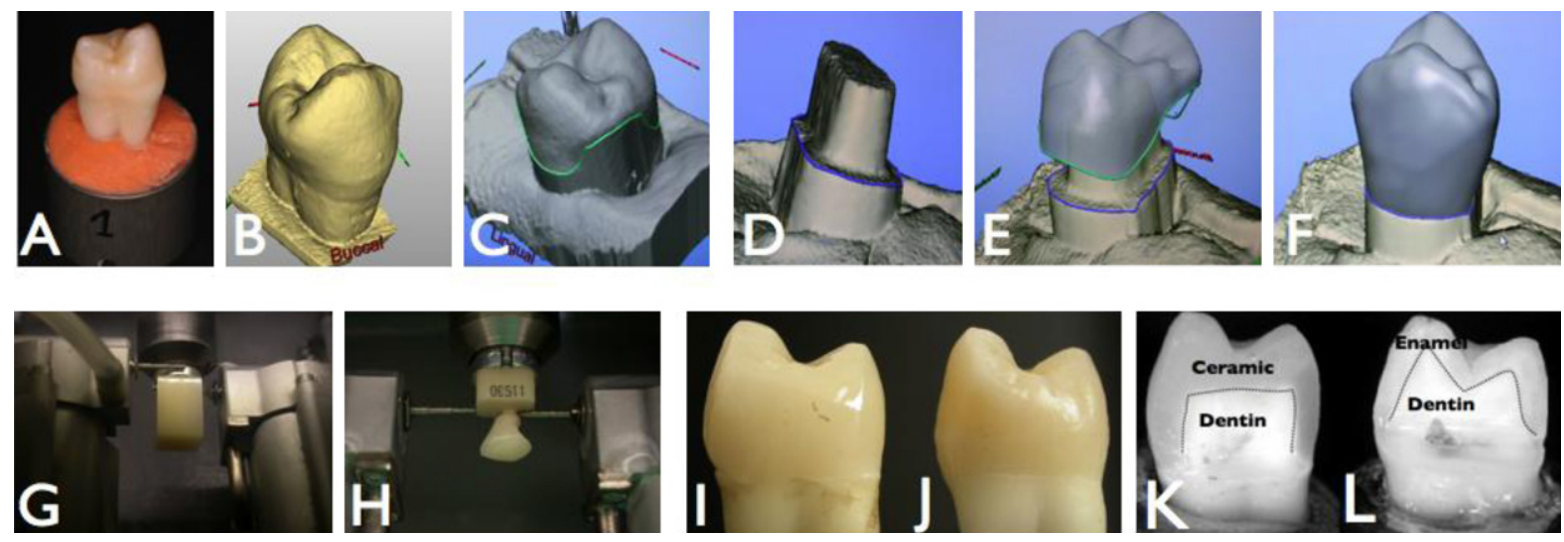

Fig. 1 - Cloning process: the intact natural crown sample (A), optical print (B), shaping (C), optical print of the second tooth prepared to receive the prosthetic clone (D), adaptation of the shaping on the tooth prepared (E), CAD finished, CAM ready (F), ceramic block in the milling unit (G), the prosthetic clone milled $(\mathrm{H})$, the prosthetic clone cemented on the tooth prepared (I), the natural tooth $(\mathrm{J})$, vertical cuts $(\mathrm{K})$, prosthetic clone $(\mathrm{L})$ natural tooth.

force can be applied to the tooth directly with the force transducer or through a relay rod. Force has always been applied to the same part of the lingual cusp. Preliminary testing by SI showed good performances of the mechanical set-up and no spurious displacements.

\subsection{SI apparatus}

The optical set-up was previously presented in detail [16]. The frequency-doubled YAG laser emits $50 \mathrm{~mW}$ at $532 \mathrm{~nm}$ wavelength in the green range. The laser beam is then injected in a COTS (commercially off-the-shelf) system (Canadian Instruments, Nottingham, UK) offering injection, variable intensity coupling in the output fibers, and phase shifting.

There are two input fibers: one for injection and one for detection of the reflected signal at the output fiber interfaces. Parts of the output fibers are bared and wrapped around piezoelectric transducers. The phase shift is applied on either or both of the two output fibers by applying a voltage at the PZT and thus generating a tiny extension of the fiber. The system is protected from thermal and mechanical effects by a plastic box and is easily breadboardable. Phase shifts were calibrated using common procedures [16].

A sensitive optical fiber in-plane interferometer has been designed with sensitivity vector Sv. Horizontal sensitivity is achieved. Two symmetrical beams produce an interference signal on the whole object and then illuminate the samples. The objects are optically rough-rendered by white powder. An XC70 CCD camera (Sony, Tokyo, Japan) records the sample surface under loading forces. The images are then stored in live memory or on the computer's hard disk.

The image processing was performed using the appropriate software in LabView, and the results are presented as vector maps or false color maps. Isodisplacement maps can be visualized in real time during the loading of the sample. The system can handle areas ranging from $5 \mathrm{~mm} \times 5 \mathrm{~mm}$ to $1 \mathrm{~m} \times 1 \mathrm{~m}$ when using the appropriate type of laser and loading system. The measurement uncertainty is approximately $50 \mathrm{~nm}$, which is common for interferometric measurement in a controlled environment. The displacement resolution is approximately $10 \mathrm{~nm}$, while the spatial resolution is directly linked to the magnification of the object scene on the image sensor $(1280 \times 900$ pixels $)$.

A "4-buckets" phase shifting algorithm leads to phase variations during the compression test [17]. During the test, the initial phase state was memory-resident and real-time subtracted from the current state. Sometimes the reference state was also refreshed because for some loading steps, the number of fringes can be too high, and the resulting noise would interfere with the interpretation of the resulting fringes. Interferograms show the in-plane displacements from phase shifting speckle interferometry. Highest quality images were stored on the hard disk and overlaid with upper jaw position and load value $(\mathrm{N})$.

\subsection{Typical experiments}

Specimens were white powdered using Eutest 3 Developer Castolin Eutectic powder (Castolin, Lausanne, Switzerland) to generate a uniform diffusing surface and to avoid different modulations between dentin and enamel or ceramic and dentin. The same loads were applied to the natural tooth and the prosthetic crown samples to allow comparisons of their respective compressive behaviors.

The starting load was approximately $0 \mathrm{~N}$. The compression was increased stepwise, performing discrete displacements of the transducer tip (one step is approximately $1.6 \mathrm{~nm}$ of Y-displacement). Therefore, a greater number of steps corresponds to a higher compression. The sample can be loaded or unloaded. The loading range applied to the different samples was between $5 \mathrm{~N}$ and $120 \mathrm{~N}$. The CCD camera records at the sample surface the interferences of the two illumination beams coming from the two output optical fibers. Live fringes are displayed between a reference state and the current load state. Between minimal and maximal loading, different phase maps are recorded and stored in the memory.

The mechanical deformations are computed from the displacement maps generated from the phase difference maps.

Speckle interferometry is a relative displacement measurement. The maximum range between two successive 


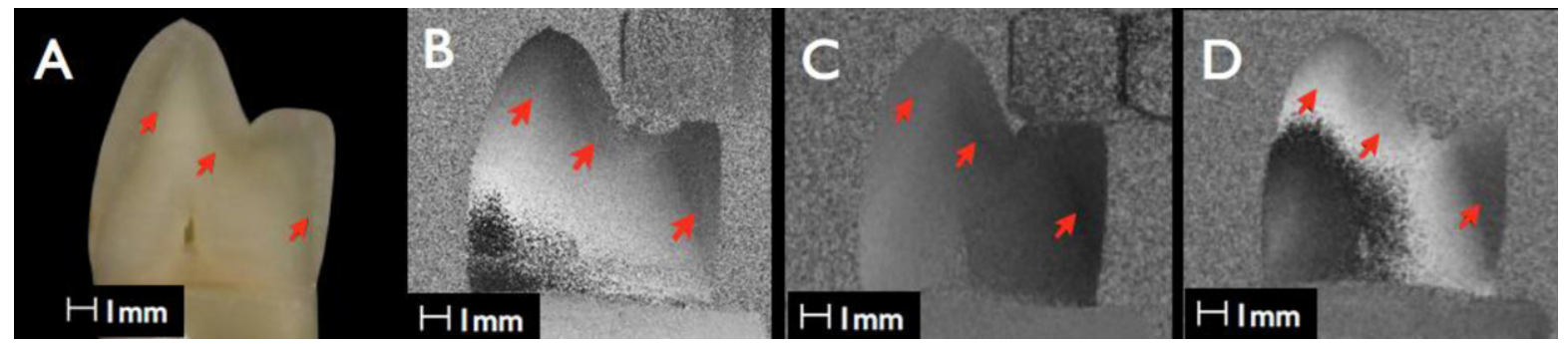

Fig. 2 - Natural tooth sample under different loads. (A) White light images. (B) Screenshot no. 04: force: $35.5 \mathrm{~N}, \Delta \mathrm{F}: 35.5 \mathrm{~N}$ (new ref: 0). (C) Screenshot no. 13: force: $40.23 \mathrm{~N}, \Delta F: 0.16 \mathrm{~N}$ (new ref: 40.39). (D) Screenshot no. 24: force: $82.54 \mathrm{~N}, \Delta \mathrm{F}: 13.96 \mathrm{~N}$ (new ref: 96.5).

measurements is approximately $20 \mu \mathrm{m}$. In our experiments, we need to record smaller steps as some mechanical noise appears. Therefore, new displacement references (zero displacement reset) are recorded during the test.

\subsection{Displacement calculations}

Different operation modes of SI are commonly used, e.g., subtraction-mode, time-averaged SI, and double-pulsed SI [16]. In this work, we focus on subtraction-mode SI, or more specifically, on phase-shifting SI, which is mainly used for static deformation measurements.

Combining the primary interference pattern phase changes between the recordings yields new secondary interference fringes (also called correlation fringes).

The variable $\varphi_{\mathrm{s}}$ denotes the start phase (also called speckle phase) at the initial state of the object. The variable $\Delta \varphi$ represents the phase change between two states.

These speckle interferograms can be subtracted and lead to the following equation for the secondary interference fringe pattern, assuming perfect spatial correlation between the two primary speckle patterns:

$I_{1}-I_{2}=2 \sqrt{I_{r} I_{\mathrm{O}}}\left(\cos \left(\varphi_{\mathrm{S}}+\Delta \varphi\right)-\cos \left(\varphi_{\mathrm{S}}\right)\right)$

Currently, noise limits the accuracy of intensity subtraction SI to approximately $15 \mathrm{~nm}$. Regarding the in-plane sensitivity of the setup, the angle of the impinging beam $\theta$ is approximately $30^{\circ}$ and determines the correspondence between the phase gray level variation and the in-plane displacement $u_{\mathrm{x}}$.
In our interferometer, the wavelength of the laser is $532 \mathrm{~nm}$, and its relationship with the in-plane displacement is given by the following equation:

$u_{\mathrm{x}}=\frac{\lambda}{4 \pi \sin \theta} \varphi_{\mathrm{x}}$

The resulting variation of one gray level of phase $u_{x}$ is approximately $2.08 \mathrm{~nm}$.

\section{Results}

Five sets of two samples each (natural tooth and prosthetic crown) were produced. The experimental protocol was tested and validated with four of them. Presented results correspond solely to the fifth sample (for natural tooth and prosthetic clone).

The interferometric images distinctly show the behavior of the samples and confirm the quality of the mechanical apparatus and the integrity of the dedicated sample holder (Figs. 2 and 3).

A natural tooth is presented in Fig. 2. Figure captions also present the screenshot number (or image number), the force applied to the sample, the force difference from the reference state, and force from the reference state (or phase reset).

Fig. 2A displays the sample under white light before painting. Fig. 2B-D is SI images recorded at different compression levels and present typical fringes. Fringes occur when the displacement induces an optical phase of $360-0^{\circ}$. The displacement in the $X$ direction is computed from the optical phase.

In Fig. 2B-D, different continuous gray lines demarcate the DEJ (red arrows) corresponding to the image taken in white

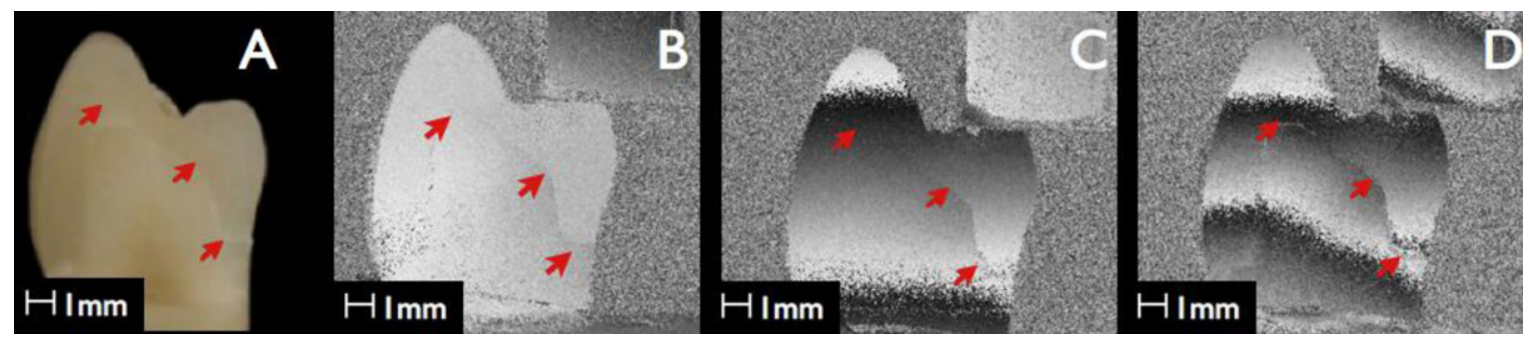

Fig. 3 - Prosthetic crown sample under different loads. (A) White light images. (B) Screenshot no. 08: force 36.78 N, $\Delta F$ : 2.57 N (new ref: $41.01 \mathrm{~N}$ ). (C) Screenshot no. 15: force 77.56 N, $\Delta F: 5.29 \mathrm{~N}$ (new ref: 82.85 N). (D) Screenshot no. 33: force 64.09 N, $\Delta F: 18.76 \mathrm{~N}$ (new ref: $41.01 \mathrm{~N}$ ). 

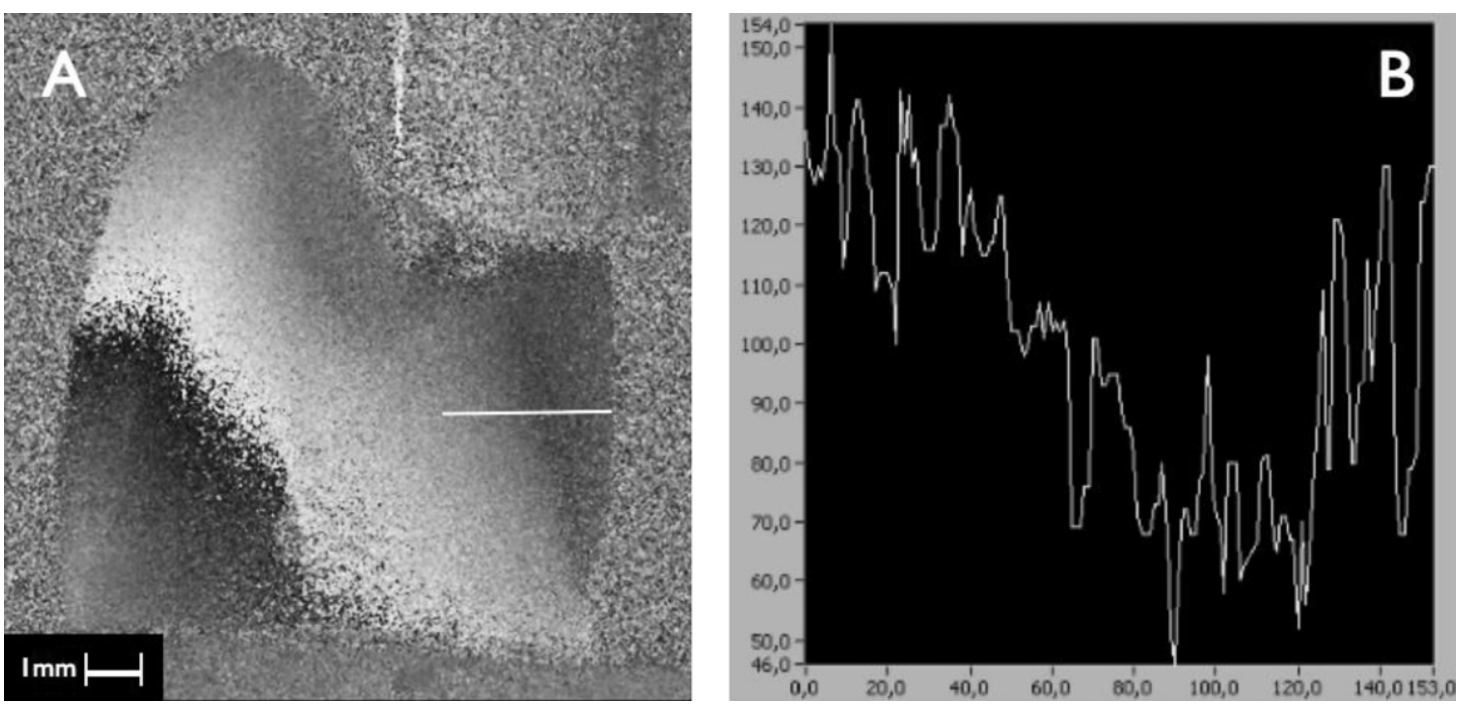

Fig. 4 - Natural tooth behavior at a load of $38.65 \mathrm{~N}, \Delta F=1.74 \mathrm{~N}$, new ref: $40.39 \mathrm{~N}$. (A) SI image: the single white line corresponds to the region of interest (ROI) used for the calculation of displacement. (B) Displacement curve along the white line, displacements in $\mathrm{nm}$ versus position in pixels. Step is about $43 \mathrm{~nm}$.

light (2A). The DEJ is clearly visible when the applied force reaches $35.5 \mathrm{~N}$. The DEJ is always more visible in front of the loading point.

Fig. 3A presents a prosthetic crown sample under white light before painting, and Fig. 3B-D are recorded at different compression levels and present typical fringes at different loads.

The DCJ appears from $38 \mathrm{~N}$ and is clearly visible in Fig. 3B-D (red arrows). For the prosthetic crowns, it appears as a continuous gray line corresponding to the image taken in white light (Fig. 3A).

The SI images displayed in Fig. 2B-D show that the natural enamel cap moves independently from the dentin. This difference is clearly delimited by a line corresponding to the anatomical location of the dentin-enamel junction (DEJ). For the prosthetic crown, in the SI images (Fig. 3B-D) the same kind of shift occurs at the cement junction of the ceramic crown with the dentin. The DEJ is less well marked than the DCJ due to the smaller associated displacements. Large variations in intensity (Fig. 3C and D) correspond to fringes resulting from rigid body motion (in plane rotation projected onto the sensitivity vector).

The highest loading values enabling the distinction of the interface zone were $117.4 \mathrm{~N}$ for the prosthetic crown and $82.5 \mathrm{~N}$ for the natural tooth. Beyond $120 \mathrm{~N}$, all samples behaved like rigid bodies. Around $200 \mathrm{~N}$, some samples were destroyed due to fractures of the brittle materials. From all of the screenshots, different images were chosen for use in computing the displacement maps.

In Fig. 4A, from left to right, we denote the transition between light gray and dark gray corresponding to the region between the dentin and the enamel. One horizontal white line has been defined as the region of interest (ROI). In Fig. 4B, the curve represents the displacement change in nanometers versus the position along the white straight line shown in Fig. 4A. The relative displacement between the dentin and the enamel is $52 \mathrm{~nm}$ for loads between $38.6 \mathrm{~N}$ and $40.4 \mathrm{~N}$ $(\Delta F=1.8 \mathrm{~N})$.

In Fig. 4B, the gray level are rising at the end of the curve as it is close to the right edge of the tooth, it is clearly visible on the native image with higher magnification. This is generated by the smooth inplane tilt of the palatine cuspid following the upper displacement of the upper jaw rod from the mechanical testing as the force decreases from $40.39 \mathrm{~N}$ to $38.65 \mathrm{~N}$.

In Fig. 5A, in front of the cusp subjected to the load, the delineation made by the DEJ is visible, and the gray levels differ because of a different accommodation.

To calculate the displacement value, six red equal parallel lines were defined perpendicularly across the DEJ. The red lines are separated by 1 pixel one to the other, and so appear as a bold red line on the figures. The displacement is the average value of the six-stacked profiles. In Fig. 5B, the black curve is the mean value fitting of the blue curve values along the six paths, and the blue curve is one of the six displacement curves. The mean relative displacement between the dentin and the enamel is about $20 \mathrm{~nm}$ for loads between $39.21 \mathrm{~N}$ and $40.39 \mathrm{~N}(\Delta F=1.18 \mathrm{~N})$.

The same analytical process was applied for the prosthetic crowns (Figs. 6-8).

In Fig. 6A, one horizontal black line was defined as the region of interest, as the technical noise was less prominent than for the natural tooth. The area of interest is located in a fringe in front of the stress zone from left to right. We denote the transition between light gray and dark gray corresponding to the region between the dentin and the ceramic cap. In Fig. 6B, the curve represents the displacement change in nanometers versus the position along the white straight line shown in Fig. 6A. The relative displacement between the dentin and the ceramic cap is $43 \mathrm{~nm}$ for loads between $82.8 \mathrm{~N}$ and $76.3 \mathrm{~N}(\Delta \mathrm{F}=6.5 \mathrm{~N})$.

In Fig. 7, to evaluate the displacement values, six equal parallel straight paths have been defined in the region of interest 

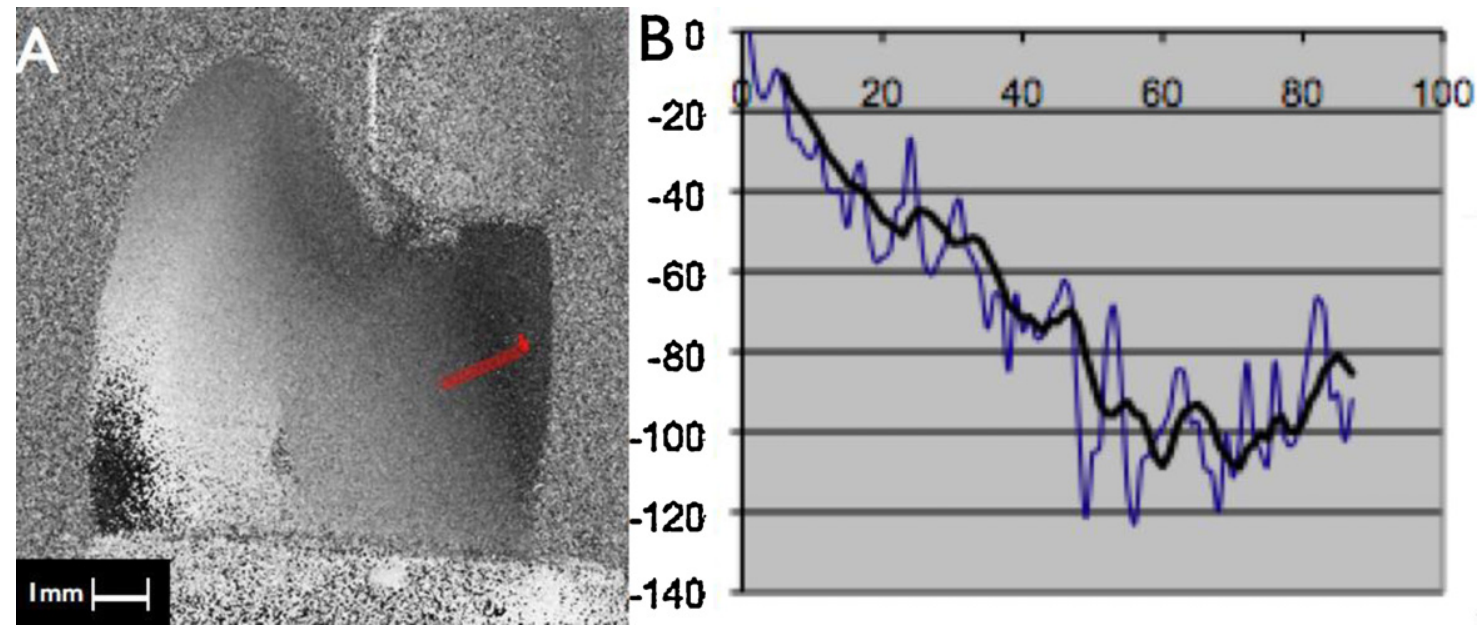

Fig. 5 - Natural tooth behavior at a load of $39.21 \mathrm{~N}, \Delta F=1.18 \mathrm{~N}$, new ref: $40.39 \mathrm{~N}$. (A) SI image: six red equal parallel lines, 1 pixel separated, are defined across the DEJ. (B) Displacement curves: blue curve: displacement values along one of the six red lines. Black bold curve is the mean value fitting of the 6 blue curves. (For interpretation of the references to color in this figure legend, the reader is referred to the web version of the article.)

across the DCJ in the palatine zone in front of the loading point (Fig. 7A). The six red lines are drawn perpendicularly to the DCJ to accurately measure the displacement variation across the DCJ. This is much appropriate than the single horizontal black line for Fig. 6 as the red lines enable the real comparison from one side to the other perpendicular to the DCJ.

In Fig. 7B, the displacement is displayed as the average value of the six stacked profiles. The black curve is the median value fitting of the blue curve values. In this case, the relative displacement is approximately $95 \mathrm{~nm}$ for loads between $82.8 \mathrm{~N}$ and $72.8 \mathrm{~N}(\Delta F=10 \mathrm{~N})$. It shows clearly the effect of the DCJ acting as an accommodation area for the applied displacement, but with a sharper slope than the natural DEJ.

The SI image in Fig. 8 was selected to represent the characteristic isodisplacement map of the discontinuity zone located in front of the loading point for a ceramic crown. A colored and zoomed image is also presented. The fringe shift in the

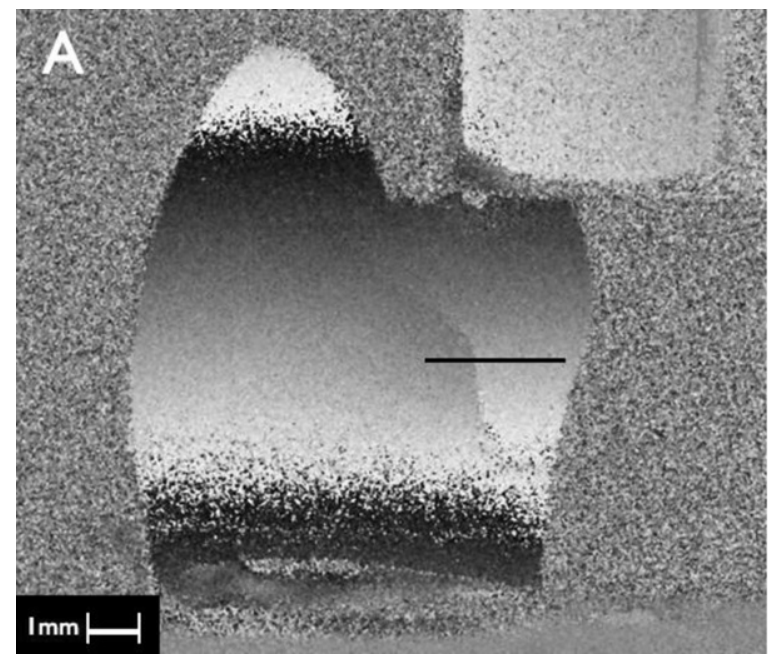

region of interest clearly delineates a mechanical interface between the ceramic and the dentin, which are separated by the cement.

Fig. 8 presents the characteristic fringes obtained for a huge load difference $(30.07 \mathrm{~N})$ and the important noise occurring due to some speckle decorrelation effect. Results from Fig. 8 show it is mandatory to make step references enabling smaller displacement load and thus very low decorrelation noise.

Fig. 9 shows the natural tooth with the same charge than prosthetic tooth (Fig. 8) and allows to compare DEJ and DCJ.

\section{Discussion}

The importance of the DEJ as an interface binding the enamel and dentin surfaces has long been recognized (Tylman [2]). Our understanding of the role and the location of the DEJ has

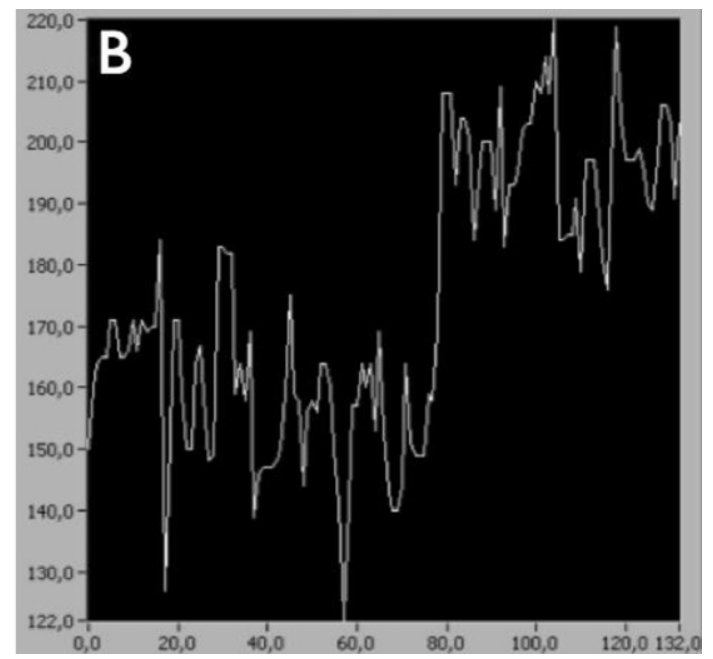

Fig. 6 - Prosthetic crown behavior at a load of $76.34 \mathrm{~N}, \Delta F=6.51 \mathrm{~N}$, new ref: $82.85 \mathrm{~N}$. (A) SI image: a single black line corresponds to the region of interest (ROI) used for displacement calculation. (B) Displacement curve along the black line, displacements in $\mathrm{nm}$ versus position in pixels. Step is about $43 \mathrm{~nm}$. 

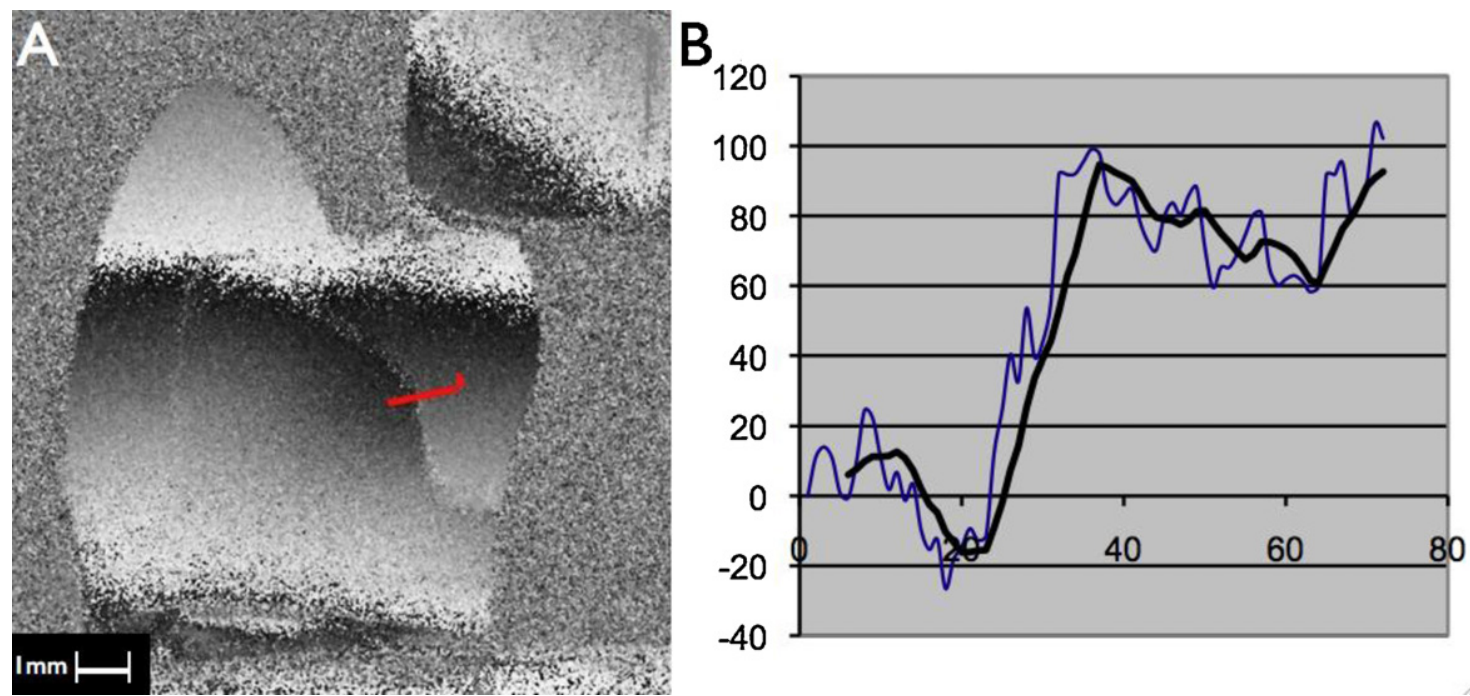

Fig. 7 - Prosthetic crown behavior under a load of $72.85 \mathrm{~N}, \Delta F=10 \mathrm{~N}$, new ref: $82.85 \mathrm{~N}$. (A) SI image: six red equal parallel lines, 1 pixel separated, are defined across the DEJ. (B) Displacement curves: blue curve: displacement values along one of the six red lines. Black bold curve is the mean value fitting of the 6 blue curves. The displacement is approximately $95 \mathrm{~nm}$. (For interpretation of the references to color in this figure legend, the reader is referred to the web version of the article.)

evolved with ongoing research. The unique mechanical properties of this zone were first recognized from micro-hardness profiles (Craig et al. [18,19]). Wang and Weiner [7] measured the strain across this zone when a compressive load was applied and suggested that this is an important working part of the tooth during mastication. An asymmetry between enamel and dentin was noted previously (Wood et al. [20]) but was not quantified. Bechtle et al. explained the phenomenon of crack arrest at the DEJ using the elastic modulus mismatch between the dentin and the enamel [21].

It was found (Zaslansky [9]) using SI that a compressive load applied to the tip of the main cusp of a human premolar caused the entire enamel cap to move essentially as a stiff body, tilting toward the buccal surface.

Recently, Barak et al. [22] highlighted the importance of enamel in a whole-tooth demonstration through a finite element model study and validated their findings by a metrology method. Chattah et al. [23] showed that the enamel cap in a minipig animal model is capable of deforming and rotating at loads as low as $16 \mathrm{~N}$. Zaslansky et al. [9] showed that the enamel cap of an isolated human premolar did not deform or rotate at loads lower than $80 \mathrm{~N}$. Human enamel is stiffer than that of the minipig, and the cusps do not deform or crack until high loads are reached. However, in both cases, the aim was to preserve the functionality of the grinding surfaces over long
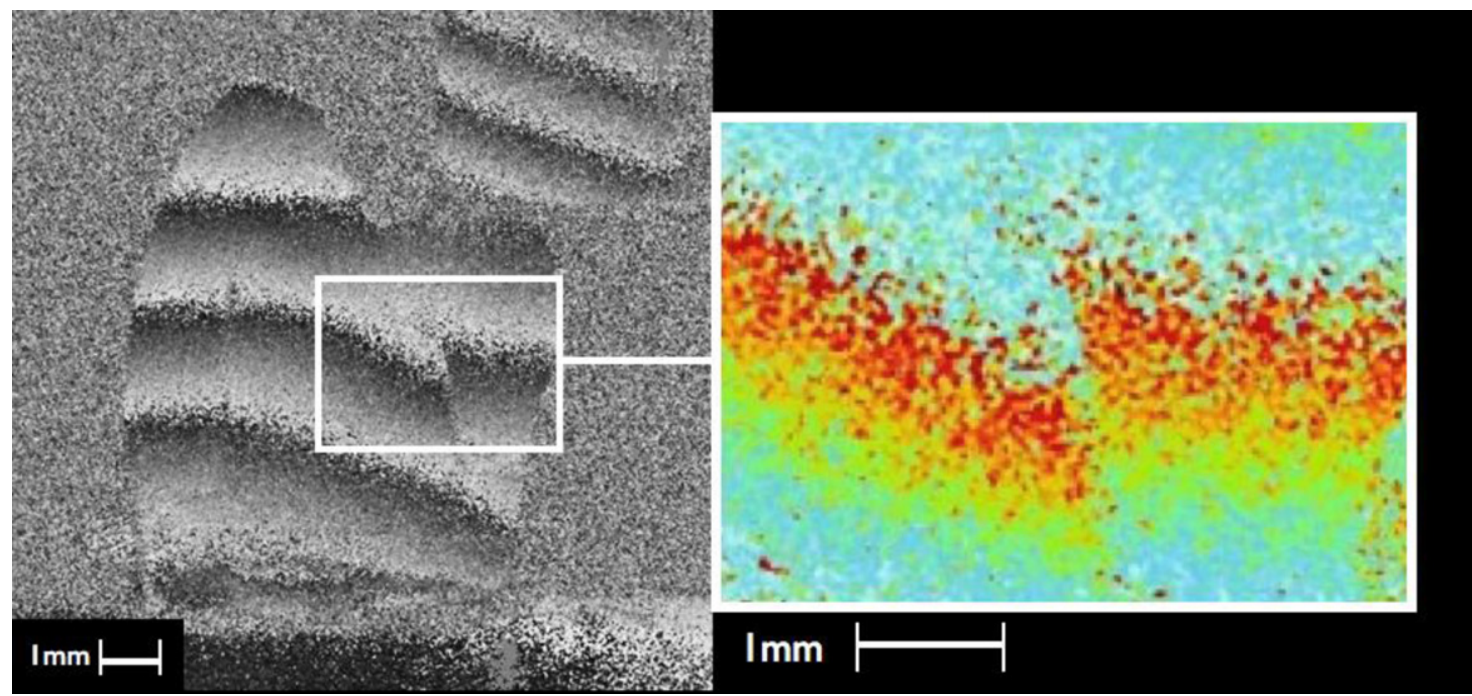

Fig. 8 - SI image sample "prosthetic crown" and displacements shown in a contour colored map. Behavior for a load of $52.78 \mathrm{~N}, \Delta F=30.07 \mathrm{~N}$, new ref: $82.85 \mathrm{~N}$. 


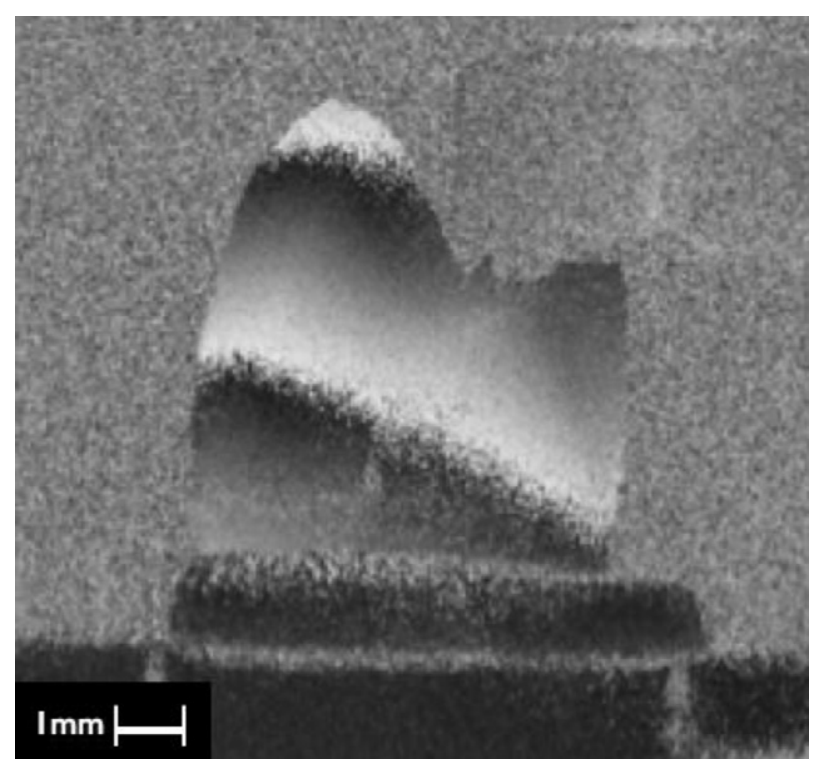

Fig. 9 - SI image sample "natural tooth". Behavior for a load of $52.3 \mathrm{~N}, \Delta F=6.5 \mathrm{~N}$, new ref: $58.8 \mathrm{~N}$.

periods of time. The use of these opposing strategies to achieve the same end has been attributed to phylogenetic differences in masticatory function. $[24,25]$. The minipig molar is capable of deforming and rotating at low loads, and the intrinsic reaction of the crown to eccentric loading is complemented and even enhanced by the structures supporting the tooth [23].

In our study, we considered the half-cut crown because this configuration has the major advantage of observing the inplane behavior of the DEJ and the DCJ in and doing so from either far from or close to the loading zone. Zaslansky worked with parallelepiped cuts from premolars [9], while Chattah et al. [23-25] studied whole teeth. On SI images, the interfaces appear distinctly along their entire lengths, thus showing their complete loading (from 35.5 N for the DEJ on the natural tooth and from $36 \mathrm{~N}$ for the prosthetic crown). The first interpretation confirms that the enamel bulk and the ceramic cap will move slightly under loading as rigid bodies. However, the mechanical response is different for the same applied loading force, and the displacement is larger for ceramic than for enamel. Thus in Fig. 8, at a load of $52.7 \mathrm{~N}$, the SI image clearly shows a relative displacement between enamel and dentin. In Fig. 9, at a similar load (52.3 N), the image identifies the DEJ and the differing behaviors of the enamel and dentin. However, it does not show a relative displacement as high as in Fig. 8. This displacement is confirmed in SI pictures in Figs. 6 and 7, with values of $83 \mathrm{~nm}$ and $95 \mathrm{~nm}$, respectively. This behavior demonstrates the accommodation strength of the DCJ and confirms its protective role for the ceramic caps.

Moreover, we emphasize that the displacement of the crown, whether enamel or ceramic, is different if the measurement area is far from or close to the loading zone. In each case, the larger displacement is located opposite to the load. The gray levels maps also show a displacement of the opposite cuspid but of smaller magnitude.

For the natural tooth, Zaslansky writes, "the asymmetry in stiffness between the buccal and lingual sides may therefore have a profound significance in determining how exactly the enamel cap responds to load during mastication". This asymmetric nature of the structure may also contribute to the distribution of loads that are not applied along the long axis of the tooth. The possible asymmetry in stiffness between the buccal and lingual sides of the tooth points to a basic property of tooth function, presumably related to the precise manner in which stress is distributed during mastication.

Conversely, for the prosthetic crown, the cement joint thickness will be the same everywhere $(100 \mu \mathrm{m})$, except in the zone of the cervical joint, where it is approximately $40 \mu \mathrm{m}$. The displacement of the ceramic cap will be a function of the thickness of the cement and its elastic modulus [26]. The behavior of the samples appears to be rational. Above a certain load (117.4 $\mathrm{N}$ for the prosthetic crown and $82.6 \mathrm{~N}$ for the natural tooth), the SI images show that the DEJ and the DCJ cannot be observed. When the interfaces in both settings can no longer accommodate the loading stress, the teeth begin to act as whole rigid objects, and higher loading forces generate cracks and fractures of the samples.

Our work tends to confirm both Zaslansky's and Weiner's studies: the DEJ zone is an important part of the tooth structure. Moreover, we have demonstrated a comparable behavior for the DCJ. We believe these zones are crucial for the stress resistance of the crown structure, whether natural or prosthetic. For monobloc vitreous ceramic prosthetic crowns, load resistance is a result of the cementing process $[27,28]$.

The soft DEJ interface is actually a graded structure, and much remains to be understood about the manner in which the whole tooth behaves under load. Figs. 6 and 7 show that the DCJ presents a graded behavior but with a sharper slope than the DEJ. An interesting use of dento-prosthetic spacing could be to select the cement joint thickness based upon the cement elastic modulus. This would refine attempts to mimic the physiological behavior of the natural tooth [29], according to the biomimetic concept [30]. This seems already very important as several authors have investigated the effect of differences in the resin-cement elastic modulus on stresstransmission to several composite or ceramic reconstructions $[31,32]$. Other authors have studied the influence of different bonding agents under stress on the internal and marginal adaptation of composite or ceramic reconstructions [33].

The biomimetic principle through the use of reconstruction ceramics that have wear coefficients close to that of natural enamel resulting from cements with an elastic modulus similar to that of the natural DEJ, we can expect the construction of real biomimetic prosthetic teeth in the near future.

\section{Conclusion}

Speckle interferometry is highly recommended for performing displacement measurements for dental biomechanics. The SI approach allows for the measurement of the mechanical properties of biological structures and other biomaterials that are a few hundred microns thick. The strain accommodation capacity of the tooth is derived from the differential displacement between the enamel and the dentin. The dentin-enamel junction acts as an interface until a certain maximal loading. Beyond this threshold, the loading accommodation property 
disappears, and the tooth then behaves as a rigid body. We demonstrate a similar behavior for the prosthetic crown tooth. In this case, the enamel is replaced by the ceramic crown and the dentin-enamel junction by the dentin-cement junction. These behaviors indicate the interface role of the DEJ and the DCJ in the capacity of teeth to accommodate the stresses of their physiological functions or even of their parafunctions. For the cement characteristics and the cement thickness used in this paper, the accommodation effect of the DCJ is less marked than that of the DEJ. Future study will deal with the effect of the cement characteristics and the cement thickness by applying SI for samples having different cements and different thickness of cement joint, thanks to the CAD/CAM system. The limits of SI measurement are the sensitivity to rigid body motion and decorrelation noise affecting the spatial resolution. This can be enhanced by using smaller pixel sensors with higher photographic magnification.

\section{R E F E R E N C E S}

[1] Imbeni V, Kruzic JJ, Marshall GW, Marshall SJ, Ritchie RO. The dentin-enamel junction and the fracture of human teeth. Nature Materials 2005;4:229-32.

[2] Tylman SD. The dentino-enamel junction. Journal of Dental Research 1928;8:615-22.

[3] Habelitz S, Marshall SJ, Marshall GW, Balooch M. The functional width of the dentino-enamel junction determined by AFM-based nanoscratching. Journal of Structural Biology 2001;135:244-301.

[4] Lin CP, Douglas WH. Structure-property relations and crack resistance at the bovine dentin-enamel junction. Journal of Dental Research 1994;73:1072-8.

[5] Meyer JM, Bodier-Houlle P, Cuisinier FJG, Lesot H, Ruch JV. Initial aspects of mineralization at the dentino-enamel junction in embryonic mouse incisor in vivo and in vitro: a TEM comparative study. In Vitro Cellular and Developmental Biology-Animal 1999;35(March (3)):159-68.

[6] Marshall GW, Balooch M, Gallagher RR, Gansky SA, Marshall SJ. Mechanical properties of the dentin-enamel junction: AFM studies of nano hardness, elastic modulus and fracture. Journal of Biomedical Materials Research 2001;54:87-95.

[7] Wang R, Weiner S. Strain-structure relations in human teeth using Moiré fringes. Journal of Biomechanics 1998;31:135-41.

[8] Zaslansky P, Shahar R, Barak MM, Friesem A, Weiner S. Tooth and bone deformation: structure and material properties by ESPI. Proceedings of SPIE 2006;6341.

[9] Zaslansky P, Friesem AA, Weiner S. Structure and mechanical properties of the soft zone separating bulk dentin and enamel in crowns of human teeth: insight into tooth function. Journal of Structural Biology 2006;153(February (2)):188-99 [Epub. 2005 December 9].

[10] Bieniek KW, Marx R. The mechanical loading capacity of new all-ceramic crown and bridge materials. Schweizer Monatsschrift fur Zahnmedizin 1994;104(3):284-9.

[11] Gonzaga CC, Cesar PF, Miranda Jr WG, Yoshimura HN. Slow crack growth and reliability of dental ceramics. Dental Materials 2011;27(April (4)):394-406 [Epub. 2010 December 24].

[12] Addison O, Sodhi A, Fleming GJ. Seating load parameters impact on dental ceramic reinforcement conferred by cementation with resin-cements. Dental Materials 2010;26(September (9)):915-21.
[13] Attia A, Kern M. Influence of cyclic loading and luting agents on the fracture load of two all-ceramic crown systems. The Journal of Prosthetic Dentistry 2004;92(December (6)):551-6.

[14] Guarda GB, Gonçalves LS, Correr AB, Moraes RR, Sinhoreti MA, Correr-Sobrinho L. Luting glass ceramic restorations using a self-adhesive resin cement under different dentin conditions. Journal of Applied Oral Science 2010;18(June (3)):244-8.

[15] Fasbinder DJ. The Cerec system: 25 years of chairside CAD/CAM dentistry. Journal of the American Dietetic Association 2010;141(June (Suppl. 2)):3S-4S.

[16] Slangen P, De Veuster C, Renotte Y, Berwart L, Lion Y. Computer-aided interferometric measurements of drift and phase shifter calibration for DSPI (digital speckle pattern interferometry). Optical Engineering 1995;34(12): 3526-30.

[17] Creath K. Phase-measurement interferometry techniques. In: Progress in optics XXVI. Elsevier Science Publisher B.V.; 1988 [Chapter 5] p. 349-393.

[18] Craig R, Peyton FA. The micro-hardness of enamel and dentin. Journal of Dental Research 1958;37:661-8.

[19] Craig R, Gehring P, et al. Relation of structure to the microhardness of human dentin. Journal of Dental Research 1959;38:624-30.

[20] Wood JD, Wang RZ, et al. Mapping of tooth deformation caused by moisture change using Moiré interferometry. Dental Materials 2003;19:159-66.

[21] Bechtle S, Fett T, Rizzi G, Habelitz S, Klocke A, Schneider GA. Crack arrest within teeth at the dentinoenamel junction caused by elastic modulus mismatch. Biomaterials 2010;31(May (14)):4238-47 [Epub. 2010 February 18].

[22] Barak MM, Geiger S, Chattah NLT, Shahar R, Weiner S. Enamel dictates whole tooth deformation: a finite element model study validated by a metrology method. Journal of Structural Biology 2009;168:511-20.

[23] Chattah LT, Shahar R, Weiner S. Design strategy of minipig molars using electronic speckle interferometry: comparison of deformation under load between the tooth mandible complex and isolated tooth. Advanced Materials 2009;21:413-8.

[24] Strait DS, Richmond BG, Spencer MA, Ross CF, Dechow PC, Wood BA. Masticatory biomechanics and its relevance to early hominid phylogeny: an examination of palatal thickness using finité-elements analysis. Journal of Human Evolution 2007;52(May (5)):585-99.

[25] Chattah NL, Kupcik K, Shahar R, Hublin JJ, Weiner S. Structure-function relation of primate lower incisor: a study of the déformation of Macaca dentition using electronic speckle pattern interferometry (ESPI). Journal of Anatomy 2011;218(1):87-95.

[26] Nakamura T, Wakabayashi K, Kinuta S, Nishida H, Miyamae M, Yatani H. Mechanical properties of new self-adhesive resin-based cement. Journal of Prosthodontic Research 2010;54(April (2)):59-64 [Epub. 2009 October 30].

[27] Attia A, Abdelaziz KM, Freitag S, Kern M. Fracture load of composite and feldspathic all ceramic CAD/CAM crowns. Journal of Prosthetic Dentistry 2006;95(February (2)): 117-23.

[28] Attia A, Kern M. Fracture strength of all ceramic crowns luted using two bonding methods. Journal of Prosthetic Dentistry 2004;91(March (3)):247-52.

[29] Urabe I, Nakajima S, Sano H, Tagami J. Physical properties of the dentin-enamel junction region. American Journal of Dentistry 2000;13(June (3)):129-35.

[30] Magne P, Belser U. Understanding the intact tooth and the biomimetic principle. In: Magne, Belser, editors. Bonded porcelain restorations in the anterior dentition: a biomimetic approach. Chicago: Quintessence Publishing Co.; 2002. p. 23-55. 
[31] Ausiello P, Rengo S, Davidson CL, Watts DC. Stress distributions in adhesively cemented ceramic and resin-composite class II inlay restorations: a 3D-FEA study. Dental Materials 2004;20(November (9)):862-72.

[32] Burke FJ, Watts DC. Effect of differing resin luting systems on fracture resistance of teeth restored with dentin-bonded crowns. Quintessence International 1998;29(January (1)):21-7.

[33] Dietschi D, Moor L. Evaluation of the marginal and internal adaptation of different ceramic and composite inlay systems after an in vitro fatigue test. Journal of Adhesive Dentistry 1999;1(1):41-56. 